\title{
Microstructure Evolution and Its Effect on Magnetic Properties of Mn-Al Alloy Fabricated via End Milling Machining Process
}

\author{
$\mathrm{J} \mathrm{Jo}^{1}$, B Vishwanadh ${ }^{12}$, MR Shankar ${ }^{3}$ and JMK Wiezorek ${ }^{1 *}$ \\ 1. Department of Mechanical engineering and materials science, University of Pittsburgh, PA, USA. \\ 2. Materials Science Division, Bhabha Atomic Research Centre, Mumbai, India. \\ 3. Department of Industrial Engineering, University of Pittsburgh, Pittsburgh, PA, USA. \\ * Corresponding author: wiezorek@pitt.edu
}

Rare earth free permanent magnets have been required for the recent growing interests on the renewable energy technology, expecting increased usage of permanent magnets. Manganese aluminum alloy near equi-atomic composition, slightly $\mathrm{Mn}$ rich, has been considered as prospective rare earth elements free permanent magnet materials [1]. However, recent progress on the developing of Mn-Al based PM materials still does not satisfy the expected performance [2]. It has been believed that the grain refinement techniques can enhance the magnetic property of Mn-Al PM materials [3]. In the present work, novel approach, end milling machining process, was applied to fabricate nanocrystalline PM materials by Severe Plastic Deformation (SPD) to improve the magnetic properties.

$\mathrm{Mn}-\mathrm{Al}$ casted alloy having composition of $\mathrm{Mn}_{54} \mathrm{Al}_{46}$ in atomic percent has been successfully produced using vacuum induction melting technique. The as-cast sample was solutionized by conducting heat treatment at $1323 \mathrm{~K}$ for 2 hours followed by water quenching to retain the single epsilon phase before further process. End milling tool equipped manual machining process with different machining parameter (tool rotation speed) was carried out to deliver particulates with uniform external shapes of micrometer size dimensions and internally nano-structured particulates assay. X-ray diffraction (XRD), Scanning electron microscopy (SEM) and Precession electron diffraction (PED) in Transmission electron microscopy were used for characterizing the structural evolution during machining process.

Detailed microstructure analysis by electron diffraction technique is required due to the compositionally invariant phase transformation behavior of epsilon and tau phase. As shown in the figure 1.(a), resulting microstructure of solutionzing heat treatment process shows only the paramagnetic epsilon phase was formed with average grain size of $\sim 100 \mu \mathrm{m}$. Precession electron diffraction under transmitted electron beam reveals significant grain refinement of the epsilon phase after machining process (b) showing average grain size of $\sim 115 \mathrm{~nm}$ and (c) showing average grain size of $\sim 35 \mathrm{~nm}$ at different machining conditions. Based on the microstructural analysis by electron diffraction imaging, it can be concluded that the machining-based particulate preparation by end-milling operations represent suitable approaches to prepare effectively internally nano-structured paramagnetic Mn-Al alloy material offering excessive nucleation sites for the phase transformation of ferromagnetic tau-phase during subsequent thermal treatments at relatively low temperatures in short times.

Detailed microstructure evolution by electron diffraction imaging after isothermal annealing of fabricated particulates and corresponding magnetic properties relations of $\mathrm{Mn}-\mathrm{Al}$ machined particulates for PM materials will be discussed [4]. 
References:

[1] JMD Coey, Scripta Materialia (2012).

[2] Z Xiang et al., J. Magn. Magn. Mater. 475 (2019), p. 479.

[3] T Saito, Journal of Applied Physics (2005).

[4] Support from NSF ENGR CMMI-1404641 is gratefully acknowledged.
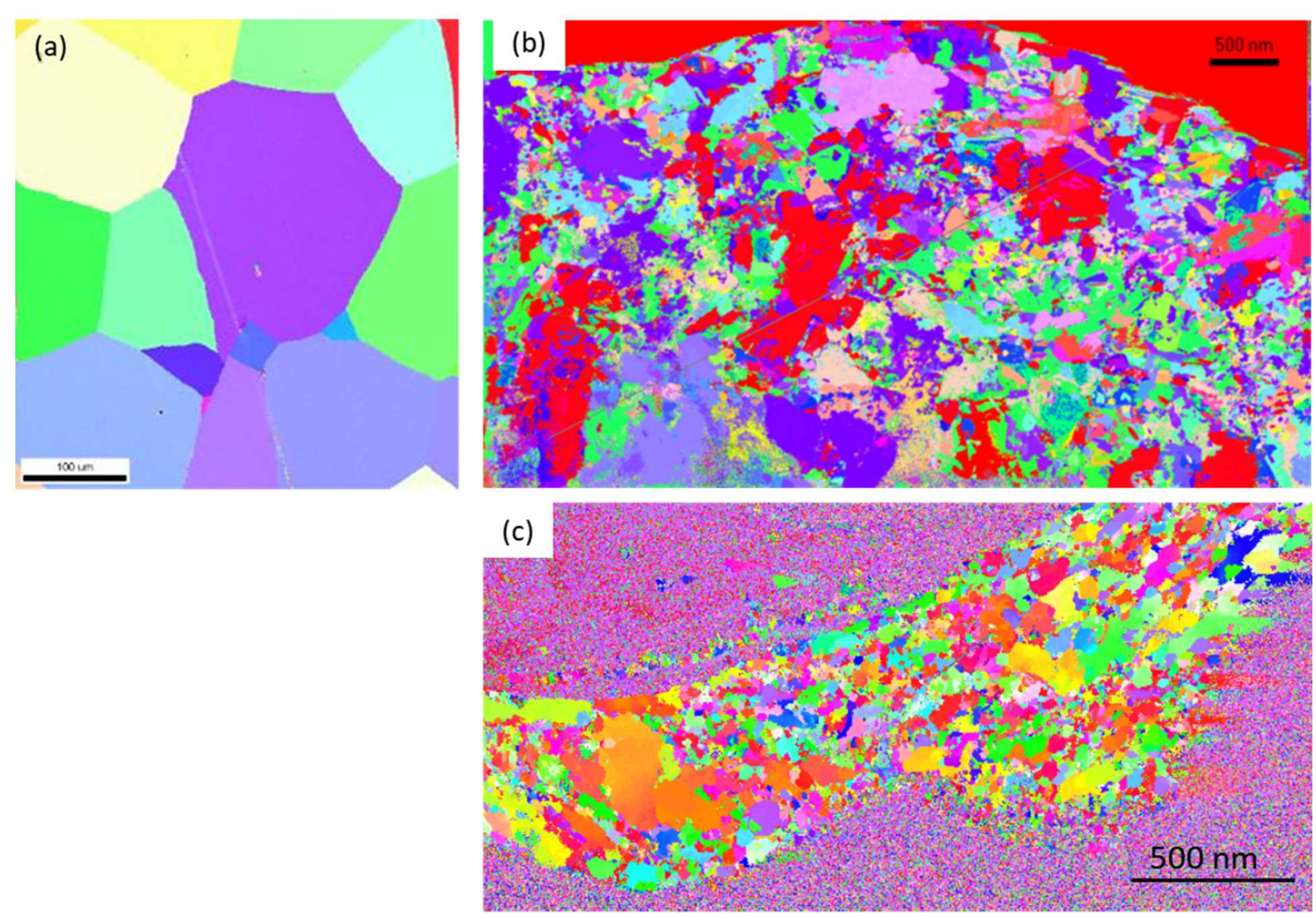

Figure 1. (a) EBSD images of bulk heat-treated epsilon phase microstructure showing average grain size of $100 \mathrm{~mm}$, (b) Inversed Pole Figure obtained from 600 RPM and (c) 3000 RPM tool rotation speed end milled particulates from PED-TEM-OIM. 